CASE REPORT

\title{
Surviving Mardi Gras in an Intensive Care Unit
}

\author{
Humaira Shafi', Allan Pablo Acosta², Roshni Sadashiv Gokhale ${ }^{3}$
}

\begin{abstract}
Penicilliosis is a life threatening fungal infection caused by Penicillium marneffei (PM). It has a high mortality rate if not picked up in time and treated appropriately. Making an early diagnosis is a key in improving outcomes. It is endemic in Southeast Asia and causes disseminated disease in the immunocompromised host especially in the human immunodeficiency virus (HIV) infected population.
\end{abstract}

We describe a HIV patient who presented with an altered sensorium. After admission he developed gastrointestinal bleeding, respiratory failure and septic shock. Blood cultures revealed yeast with high suspicion for PM. Intravenous amphotericin B was promptly initiated resulting in marked improvement of overall condition.

The learning objective of this report is to illustrate the increasing variability of clinical manifestations and absence of "typical" skin lesions making an early diagnosis difficult. In endemic regions the immunocompetent are equally susceptible. A high index of suspicion, early recognition and treatment is required to improve patient outcomes. $J$ Microbiol Infect Dis 2017; 7(1): 46-50

Keywords: Penicillium marneffei, penicilliosis, disseminated fungal infection, HIV, gastrointestinal bleeding

\section{CASE REPORT}

A 42-year-old Chinese male patient presented with fever and altered mental state five days after initiation of antiretroviral therapy. He was recently diagnosed with HIV. Risk factor was unprotected heterosexual behavior. The absolute CD4 count was 7 cells $/ \mu \mathrm{L}$ and HIV viral load was 5.0 logs. Baseline genotype resistance testing did not show resistance mutations. He was started on anti-retroviral treatment (ART) with lamivudine, tenofovir, and raltegravir.

Past medical history was significant for weight loss and poor appetite for 3 months, evaluation of which confirmed HIV. Patient declined further investigations.

Travel history was significant for travel to Vietnam, Xiamen, China and frequent visits to Malaysia. He was married, did not smoke, drink alcohol or use recreational drugs. He did not keep any pets. He was currently unemployed but previously worked as a construction manager. Family history was not significant.
A review of symptoms revealed no headache, neck stiffness, photophobia, cough, runny nose, abdominal pain, diarrhea, vomiting or skin rash.

Physical exam showed a temperature of $37^{\circ} \mathrm{C}$, heart rate of $114 \mathrm{bpm}$, respiration of 20 per min, blood pressure of $117 / 81 \mathrm{~mm} \mathrm{Hg}$ and oxygen saturation of $100 \%$ on room air. He was agitated and had auditory hallucinations. He was disoriented to time, place and person. Glasgow Coma Scale (GCS) was 14 (E4, V4, M6). There were no sign of meningeal irritation. Examination of the lungs, heart, abdomen, extremities, and lymphatic system was unremarkable. No skin rash or lesions were present.

Investigations upon admission showed hypokalemia, hyponatremia, hypoalbuminemia and anemia (Table 1).

A provisional diagnosis of central nervous system (CNS) infection was made and empirical intravenous ceftriaxone, acyclovir, and ampicillin were initiated pending cerebrospinal fluid (CSF) and blood culture results.

\footnotetext{
${ }^{1}$ Department of General Medicine, Division of Infectious Diseases, Changi General Hospital, Singapore

${ }^{2}$ Department of General Medicine, Division of Infectious Diseases, Changi General Hospital, Singapore

${ }^{3}$ Department of Respiratory and Critical Care Medicine, Changi General Hospital, Singapore

Correspondence: Dr. Humaira Shafi, Changi General Hospital, 2 Simei Street 3, Singapore, Email: humaira_shafi@cgh.com.sg Received: 18 July 2016, Accepted: 20 November 2016

Copyright (C JMID / Journal of Microbiology and Infectious Diseases 2017, All rights reserved
} 
Table 1. Laboratory investigations.

\begin{tabular}{lc}
\hline Variables & Results \\
\hline Serum Sodium & $128 \mathrm{mmol} / \mathrm{L}$ \\
Serum Potassium & $3.3 \mathrm{mmol} / \mathrm{L}$ \\
Urea & $2.2 \mathrm{mmol} / \mathrm{L}$ \\
Glucose & $5.9 \mathrm{mmol} / \mathrm{L}$ \\
Creatinine & $58 \mathrm{umol} / \mathrm{L}$ \\
Bicarbonate & $22 \mathrm{mmol} / \mathrm{L}$ \\
Magnesium & $0.77 \mathrm{mmol} / \mathrm{L}$ \\
Corrected Calcium & $2.13 \mathrm{mmol} / \mathrm{L}$ \\
Phosphate & $0.45 \mathrm{mmol} / \mathrm{L}$ \\
Albumin & $28 \mathrm{~g} / \mathrm{L}$ \\
Alkaline Phosphatase & $89 \mathrm{U} / \mathrm{L}$ \\
Alanine Transaminase & $79 \mathrm{U} / \mathrm{L}$ \\
Aspartate Transaminase & $141 \mathrm{U} / \mathrm{L}$ \\
Procalcitonin & $5.60 \mathrm{\mu g} / \mathrm{L}$ \\
CRP & $99.6 \mathrm{mg} / \mathrm{L}$ \\
Absolute CD4 & $7 \mathrm{cells} / \mathrm{LL}$ \\
Hemoglobin & $8.7 \mathrm{~g} / \mathrm{dL}$ \\
WBC & $4.7 \times 10^{3} / \mathrm{UL}$ \\
Platelets & $218 \times 10^{3} / \mathrm{UL}$ \\
CMV quantitative PCR & Not detected \\
(blood) & \\
Serum Cryptococcal Antigen & negative \\
Histoplasma antibody & Not detected \\
Stool C difficile PCR, ova \& & Not detected \\
parasite, cryptosporidia, & \\
cyclospora and isospora. & \\
Variables & Results \\
Serum Sodium & $128 \mathrm{mmol} / \mathrm{L}$ \\
\hline
\end{tabular}

Lumbar puncture showed CSF cell count 0 unit $/ \mathrm{mm}^{3}$, RBC 0 unit $/ \mathrm{mm}^{3}$, glucose $3.2 \mathrm{mmol} / \mathrm{L}$ (blood glucose $5.9 \mathrm{mmol} / \mathrm{L}$ ), protein $0.14 \mathrm{~g} / \mathrm{L}$. Gram stain was negative. AFB smear was negative. Fungal microscopy was negative. CSF VDRL and cryptococcal $\mathrm{Ag}$ were negative. Neurotropic viruses were not isolated. Noncontrasted computerized tomography (CT) brain showed a mixed lucent/sclerotic lesion with associated extra-axial soft tissue component in the left frontal region. Magnetic resonance imaging (MRI) brain with contrast confirmed a left frontal bone intra-osseous lesion associated with small extra-axial soft tissue component abutting the underlying brain parenchyma (Figure 1).
On the next day, he developed an acute significant lower Gl bleeding causing hemodynamic instability. This was treated with intravenous fluid resuscitation and blood transfusions. A CT scan of the abdomen was suspicious for active bleeding with the suggestion of colitis, and the incidental finding of tree-in-bud configuration and bilateral basal consolidation of the lung.

At this point the patient developed desaturation due to combination of consolidation and fluid overload. He was transferred to medical intensive care unit (MICU) for mechanical ventilation.

Meanwhile the CSF cultures were reported negative for bacterial infection. Ampicillin and acyclovir were discontinued. Antibiotics were switched empirically to intravenous meropenem and pentamidine to cover for sepsis secondary to hospital acquired pneumonia and presumptive Pneumocystic jiroveci (PCJ) pneumonia. Intravenous ganciclovir was also initiated for suspected CMV colitis.

Mesenteric embolization was not performed as a bleeding vessel could not be visualized on angiography. He was deemed an unsuitable candidate for endoscopy or surgical intervention due to unstable hemodynamics.

During his stay in the MICU he underwent a diagnostic bronchoscopy. Sputum and bronchoalveolar lavage (BAL) was negative for PCJ PCR, respiratory virus multiplex PCR, TB PCR, melioidosis PCR and aerobic cultures.

Within 96 hours of admission, preliminary blood cultures were reported positive for PM. Antimicrobial regimen was changed to intravenous amphotericin B. Pentamidine, meropenem and ganciclovir were then discontinued. Sputum and BAL fungal cultures subsequently also isolated PM. Patient improved markedly after commencement of intravenous amphotericin and was successfully extubated. There were no further episodes of lower gastrointestinal (GI) bleeding. He was given 18 days of intravenous amphotericin $B$ followed by oral itraconazole $200 \mathrm{mg} \mathrm{BD}$ for 7 months. He made full recovery and was discharged home in stable condition.

At the time of writing this report, he is on maintenance treatment with oral itraconazole 
$200 \mathrm{mg}$ daily and ART. He is virologically suppressed and absolute CD4 count is 38 cells $/ \mu \mathrm{L}$.

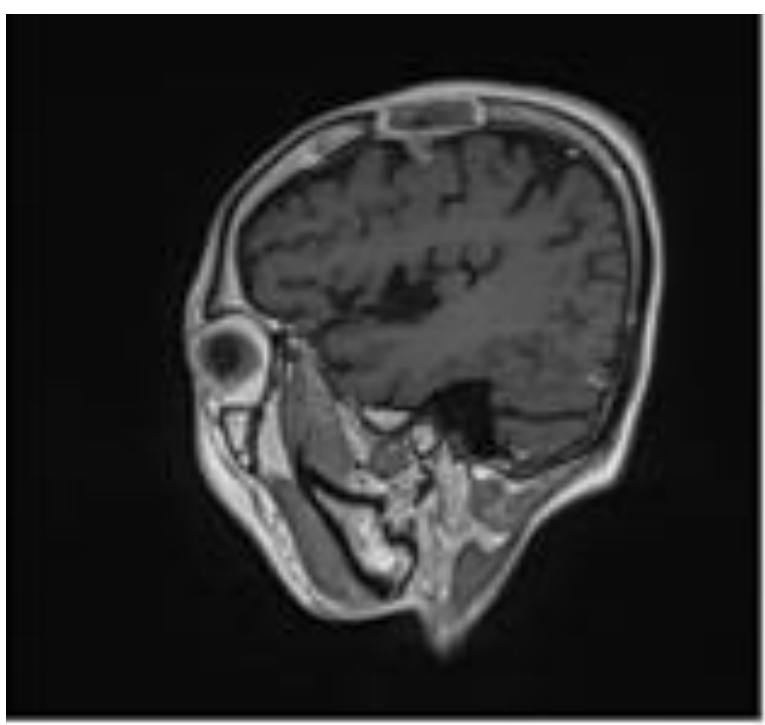

Figure 1. MRI image of the patient.

He remains on outpatient infectious disease follow up. There had been no recurrence of Gl bleeding. For the skull lesion, CT brain was repeated at a 3-month interval which showed a stable mixed sclerotic and lucent lesion in the left frontal bone. Unfortunately, patient defaulted follow up with neurosurgery.

\section{DISCUSSION}

Penicilliosis (now known as Talarmyces marnefeii) is the third most common opportunistic infection in South East Asia [1]. However when an ICD code search was carried out for the diagnosis of "penicilliosis" or "Penicillium marneffei infection", this patient was the only case found in our 1000-bed teaching hospital in last 10 years.

This case is important as it illustrates an increasing frequency of atypical presentations of penicilliosis. Our patient presented with an altered mental status with the quick onset of florid GI bleeding and the absence of "typical" skin lesions. In addition a sclerotic-lucent osseous lesion was seen on the CT brain however CSF culture was negative. His management included an attempt to empirically cover for suspected concomitant opportunistic infections in view of his critical condition until the culprit infection was identified. These were the features that made it a diagnostic and management dilemma [2-4]. We postulate that a favorable outcome in this case was due to an early diagnosis made via fungal blood cultures along with prompt initiation of antifungal treatment. Mortality has been reported to be as high as 97 percent with delay in diagnosis or treatment [5].

The only previous case series published from Singapore was in the 1999 and described 5 patients, all with HIV infections who had recently visited or were immigrants from endemic regions [6]. GI bleeding as part of symptom complex has been described rarely $[7,8]$. Ko et al. published 3 cases with AIDS where intestinal pencilliosis was presenting complaint. Additionally 4 cases were also described in this report where diagnosis was established on histopathology and intestinal cultures [2]. Le et al. from Vietnam has described CNS infection with isolation of $P M$ in CSF samples in 20 out of $21 \mathrm{HIV}$ patients [9]. A case series from South China describes the rare occurrence of osteolytic lesions in HIVnegative patients [10].

Microbiologically PM is the only one in its species to exhibit thermal dimorphism allowing it easy host colonization. It exists as yeast at a temperature of over $37^{\circ} \mathrm{C}$ and as a mould at 30 ${ }^{\circ} \mathrm{C}$ and below. The yeast form exhibits characteristic central septation and replicates by fission. In the filamentous form it grows on Sabouraud Dextrose Agar as greenish colonies which produce a diffusible red pigment.

There is little substantial information on the exact epidemiology of PM. The only two known natural reservoirs are bamboo rats and humans. It remains unclear whether the bamboo rats are only a carrier for this fungus, whose actual reservoir is the soil. It has been observed that the incidence rises with the onset of the rainy season. Mode of transmission is postulated through the respiratory route. The fungus then disseminates via the blood vessels and lymph tissue causing a systemic fungemia. Endemic areas are South East Asia which includes North east India, Vietnam, Cambodia, Thailand, Hong Kong and Southern China. Typical patient will be an immunocompromised host. Incidence is higher in HIV patients with CD4 counts $<100$. It is also found in foreigners, travelers and immigrants from the endemic areas. Common 
coexistent opportunistic infections are with mycobacterium and cryptococcus.

Clinical presentation commonly includes fever, weight loss, reticuloendothelial involvement, lymphadenopathy, hepatosplenomegaly and molluscum-like skin lesions. The literature shows that osteolytic lesions are more commonly seen in immunocompetent patients [10].

Occasionally immune reconstitution inflammatory syndrome (IRIS) and acute abdomen might also be presentations of this infection [11,12]. Anemia, pancytopenia and transaminitis are commonly found on routine blood tests.

Differential diagnosis for penicilliosis includes:

1) Fungal infections- Histoplasmosis, Cryptococcosis, $P C J$.

2) Mycobacterial infections.

3) Viral infections- cytomegalovirus.

4) Bacterial infections- Melioidosis, severe community acquired bacterial pneumonias.

Diagnosis is established by direct examination and fungal culture of blood, bone marrow (BM), tissue, BAL, pleural fluid, peritoneal fluid and CSF. Pickup rate from BM culture (100\%), skin biopsy $(90 \%)$ and blood cultures $(76 \%)$ were evaluated. Galactomannan can be falsely positive for PM. The sensitivity and specificity of serum galactomannan test for PM is $95.8 \%$ $(23 / 24)$ and $90.9 \%(30 / 33)$, respectively (cut-off index $=1.0$ ) [13]. PCR has been used only in clinical trials [14].

$P$. marneffei inh-ELISA is a promising new assay for the rapid diagnosis of PM as well as a tool for evaluating clinical response and clearance of the fungus during treatment [15].

Treatment is with intravenous amphotericin B $(0.6-1 \mathrm{mg} / \mathrm{kg} / \mathrm{d})$ for 2 weeks followed by oral itraconazole $(400 \mathrm{mg} /$ day $)$ for 10 weeks. Secondary prophylaxis with itraconazole is prescribed at a dose of $200 \mathrm{mg} /$ day till CD4 counts $>100$ or for at least 6 months (else high relapse). IRIS can occur with PM but current belief is that it should not delay ART.

In summary, PM is third most common AIDS defining illness in endemic areas. It has a wide variety of presentations and can equally affect the immunocompetent in endemic areas. High index of suspicion, establishing an early diagnosis and an early institution of antifungal therapy can save lives. Absence of skin lesions does not exclude penicilliosis.

Declaration of conflicting interests: The authors declared no conflicts of interest with respect to the authorship and/or authorship of this article.

Funding: The authors received no financial support for the research and/or authorship of this article.

Written consent was obtained from the patient for MRI image to be published in this journal.

\section{REFERENCES}

1. Duong TA. Infection due to Penicillium marneffei, an emerging pathogen: review of 155 reported cases. Clin Infect Dis 1996; 23(1):125-30.

2. $\mathrm{Ko} \mathrm{Cl}$, Hung $\mathrm{CC}$, Chen MY, Hsueh PR, Hsiao $\mathrm{CH}$, Wong JM. Endoscopic diagnosis of intestinal penicilliosis marneffei: report of three cases and review of the literature. Gastrointest Endosc 1999; 50 (1):111-4.

3. Wong SY, Wong KF. Penicillium marneffei Infection in AIDS. Patholog Res Int 2011; 2011:764293.

4. Qiu Y, Zhang J, Liu G, et al. Retrospective analysis of 14 cases of disseminated Penicillium marneffei infection with osteolytic lesions. BMC Infect Dis 2015;15:47.

5. Supparatpinyo K, Khamwan C, Baosoung V, Nelson KE, Sirisanthana T. Disseminated Penicillium marneffei infection in southeast Asia. Lancet 1994; 344(8915):110-3.

6. Kurup A, Leo YS, Tan AL, Wong SY. Disseminated Penicillium marneffei infection: a report of five cases in Singapore. Ann Acad Med Singapore 1999; 28(4):605-9.

7. Leung R, Sung JY, Chow J, Lai CK. Unusual cause of fever and diarrhea in a patient with AIDS. Penicillium marneffei infection. Dig Dis Sci 1996; 41(6):1212-5.

8. Tsui WM, Ma KF, Tsang DN. Disseminated Penicillium marneffei infection in HIV-infected subject. Histopathology 1992; 20(4):287-93.

9. Le T, Huu Chi N, Kim Cuc NT, et al. AIDSassociated Penicillium marneffei infection of the central nervous system. Clin Infect Dis 2010; 51(12):1458-62.

10. Liu GN, Huang JS, Zhong XN, et al. Penicillium marneffei infection within an osteolytic lesion in an HIV-negative patient. Int $\mathrm{J}$ Infect Dis 2014;23:1-3.

11. Ghalige HS, Sahoo B, Sharma S, Devi KR, Singh Th SC. Acute Abdomen Due to Penicillium marneffei: An Indicator of HIV Infection in Manipur State. J Clin Diagn Res 2014; 8(9):ND05-6. 
12. Liu X, Wu H, Huang X. Disseminated Penicillium marneffei infection with IRIS. ID Cases 2015;2(4):92-3.

13. Huang $Y T$, Hung $C C$, Liao $C H$, Sun HY, Chang SC, Chen YC. Detection of circulating galactomannan in serum samples for diagnosis of Penicillium marneffei infection and cryptococcosis among patients infected with human immunodeficiency virus. J Clin Microbiol 2007; 45(9):2858-2862.

14. Zheng J, Gui X, Cao Q, et al. A Clinical Study of Acquired Immunodeficiency Syndrome Associated Penicillium marneffei Infection from a NonEndemic Area in China. PLoS One 2015; 10(6):e0130376.

15. Prakit K, Nosanchuk JD, Pruksaphon K, Vanittanakom N, Youngchim S. A novel inhibition ELISA for the detection and monitoring of Penicillium marneffei antigen in human serum. Eur J Clin Microbiol Infect Dis 2016; 35 (4):647-56. 